\title{
Resonance of Open Ring Frequency Selective Surface Arrays
}

\author{
Xiao-kun Chen ${ }^{1 *}$, Jia-ao $\mathrm{Yu}^{2}$ \\ ${ }^{1}$ College of Science, Huazhong Agricultural University, Wuhan, Hubei Province 430070, P. R. \\ China. ) \\ ${ }^{2}$ Early Warning Academy, Wuhan 430019, P. R. China. \\ ${ }^{*}$ Corresponding author
}

Keywords: Frequency Selective Surface, Resonance.

\begin{abstract}
In this paper, the resonance of open ring frequency selective surface arrays is studied in particular. The new resonant cells are greatly reduced in dimensions and very compact in structures, so it is possible to realize the lower frequency selective surface. Simulation and measurement are basically in agreement.
\end{abstract}

\section{Introduction}

Frequency Selective Surfaces (FSSs), which have the band pass or band stop characteristics, find widespread applications as filters for microwaves in radomes and sub reflectors. These surfaces comprise periodically arranged resonant cells in two directions. The resonant cell may be metallic patch element or aperture element within a metallic screen[1-2]. In this paper, FSSs with a new resonant cell-open ring are introduced and the resonant characteristics are analyzed in detail..

\section{The Finite Element Model[3]}

Figure1 presents the finite element model. Due to the periodicity of the structure, a unit cell is cut out and analyzed. Periodic boundary conditions are also presented in Figure1.

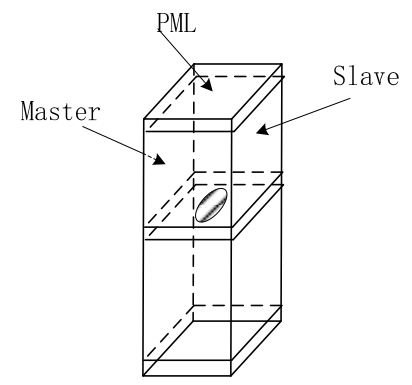

Figure 1 The finite element model.

It is common to call the surface with free variables the "master" boundary and the surface with constrained variables the "slave" boundary. The tangential component of the electric field on slave boundaries can be expressed in terms of the tangential component of the electric field on master boundaries as

$$
\vec{E}_{\text {slave }}=e^{j \phi} \vec{E}_{\text {master }}
$$

where $\phi$ is the phase angle between the master and slave boundaries and can be expressed by the incident angles of the incident wave. An incident plane wave impinges at an angle to the surface. 
PML' $\mathrm{s}$ were used on both sides of the unit cell. The functional being solved by the finite element method is :

$$
\begin{aligned}
F= & \int_{\Omega}\left(\nabla \times \vec{E}^{s^{*}} \bullet\left[\mu_{r}\right] \nabla \times \vec{E}^{s}-k_{0}{ }^{2} \vec{E}^{s^{*}} \bullet\left[\varepsilon_{r}\right] \vec{E}^{s}\right) d \Omega- \\
& \int_{\Omega}\left(k_{0}{ }^{2} \vec{E}^{s^{*}} \bullet \operatorname{ind}(P M L)\left(\left[\varepsilon_{r}\right]-\left[\mu_{r}\right] \vec{E}^{i n c}\right) d \Omega+\right. \\
& j \omega \mu_{0} \int_{\Omega} \vec{E}^{s^{*}} \bullet \operatorname{ind}(P M L)\left(\operatorname{grad}\left[\mu_{r}\right] \times \vec{H}^{i n c}\right) d \Omega
\end{aligned}
$$

Where $\vec{E}^{s}$ is the scattered field, $\vec{E}^{i n c}$ is the incident field and

$$
\text { ind }(P M L)= \begin{cases}0 & \text { in } P M L \\ 1 & \text { else }\end{cases}
$$

The calculation of the transmission coefficient is based on the ratio of the transmitted and incident power as:

$$
\text { Transmission }=10 \times \log \left(1-\frac{\int_{s_{\text {out }}} \operatorname{Re}\left(\vec{E}^{s} \times \vec{H}^{s^{*}}\right) \bullet \vec{n} d s}{\int_{S_{\text {in }}} \operatorname{Re}\left(\vec{E}^{\text {inc }} \times \vec{H}^{\text {inc }}\right) \bullet \vec{n} d s}\right)
$$

\section{Design Guidelines}

Three proposed resonant cells are realized on the substrate, $\varepsilon_{r}=3.0$ and $h=0.5 \mathrm{~mm}$. The layout and parameters of these structures were presented in Figure 2. The cells are separately closed ring, open ring with a 100 cut and open ring with a 300 cut, corresponding to Figure 2(a), (b), (c). All the cells are closed packed on square lattices and the period is $12 \mathrm{~mm}$. The inner radius is $4.5 \mathrm{~mm}$ and the outer one is $5.5 \mathrm{~mm}$.

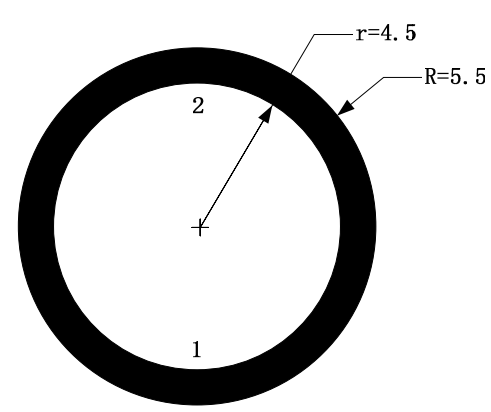

(a)

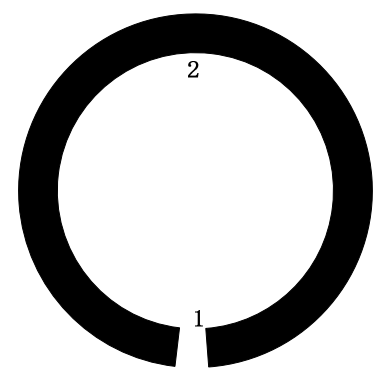

(b)

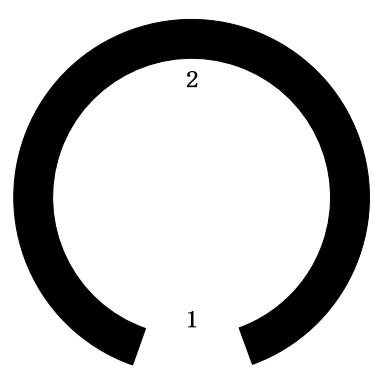

(c)

Figure 2 The shapes of resonant cells: (a) no cut (b) $10^{\circ}$ cut (c) $30^{\circ}$ cut (units: mm) 


\section{Simulation and Experimental Results}

Figure 3 shows the transmission coefficient of figure 2(a) and 2(b) at normal incidence. It is evident that when the incident $\vec{E}$ field is parallel to the cut (TM wave incident), the open ring has dual resonant frequencies and the lower one is about half of the closed ring's resonant frequency; on the contrary, when the incident $\vec{E}$ field is perpendicular to the cut (TE wave incident), the differences of the frequency response between open ring and closed ring are very small.

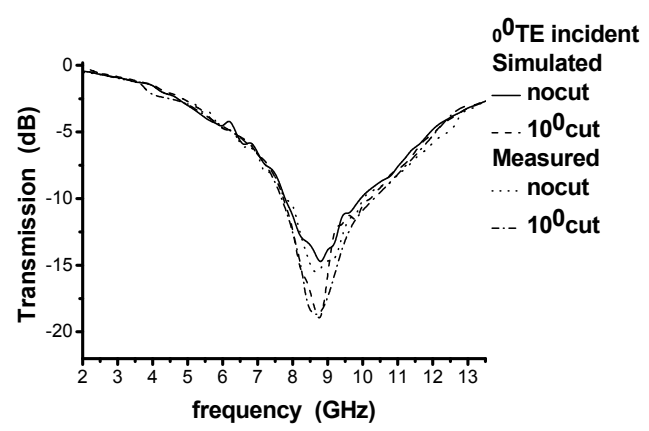

(a)

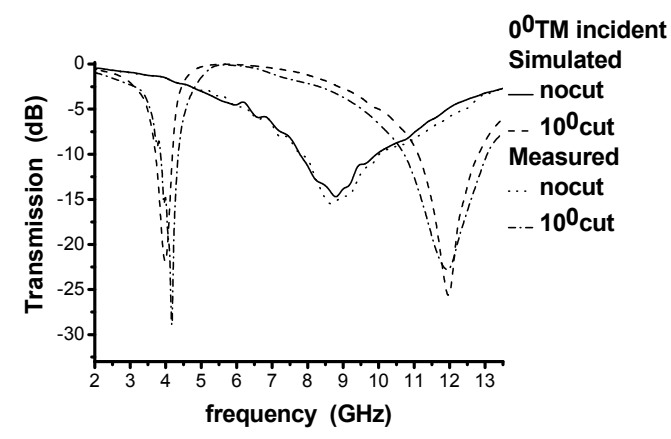

(b)

Figure 3 The transmission coefficient of figure 1(a) and 1(b) at normal incidence: (a) TE incident (b) TM incident.

The above differences between open ring and closed ring can be explained by the distribution of the induced current around the element: for closed ring, the element is symmetrical, so the response is unaltered no matter what wave (TE or TM) incident. But the current has larger differences at the points 1 and 2 for the different polarization: for TE wave incidence, the current has nulls at the points 1 and 2, so breaking the ring at 1 has almost no effect on the transmission response; for TM wave incidence, the current has maxima at the points 1 and 2, so an important change occurs when the element is cut at 1 and the element resonants in a mode with a current null at 1 only, increasing the resonant wavelength by almost a factor of 2 .

Figure 4 reveals the open element has favourable dual band and polarization selective characteristics even at 450 inclined incidence.

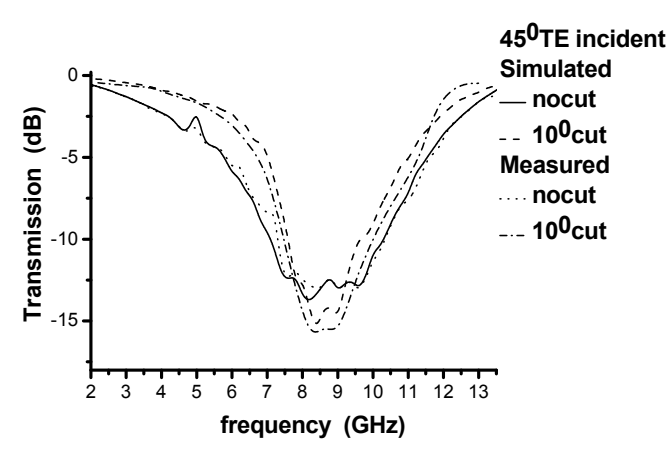

(a)

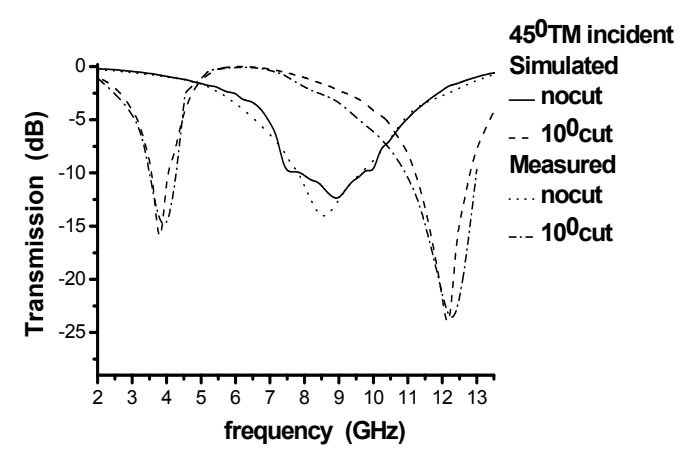

(b)

Figure 4 The transmission coefficient of figure 1(a) and 1(b) at $45^{\circ}$ inclined incidence: (a) TE incident (b) TM incident.

Figure 5 shows the transmission coefficient of figure 2(b), (c). It is evident that the larger is the cut's size, the shorter is the resonant wavelength and the higher is the resonant frequency. 


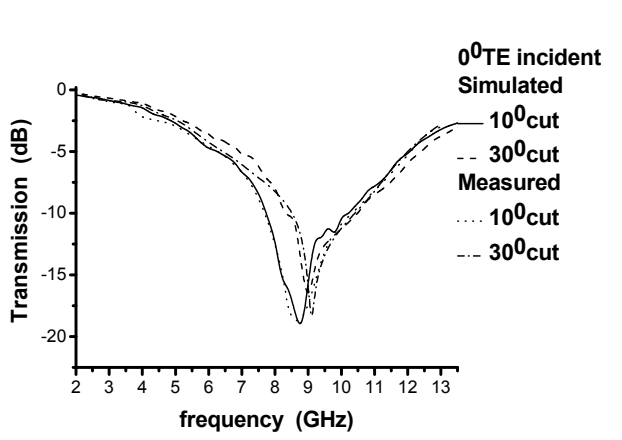

(a)

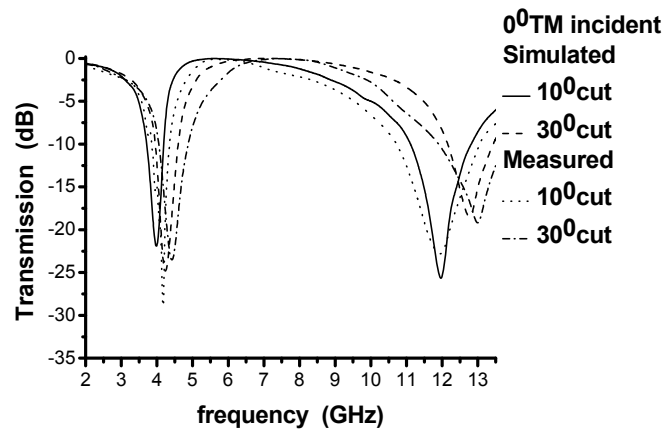

(b)

Figure 5 The transmission coefficient of figure1(b) and 1(c) at normal incidence: (a) TE incident (b) TM incident.

\section{Conclusion}

FSSs, which comprise loop element by the simple process of breaking the conductor at suitable locations, generate a large reduction in the resonant frequency, and realize dual band and polarization selection for a fixed unit cell size. This paper has important reference value to the manufacture of FSSs.

\section{References}

[1] BEN A. MUNK.FREQUENCY SELECTIVE SURFACE Theory and Design. (Wiley,New York,2001).

[2] H. Contopanagos, L. Zhang and N. Alexapoulos. Thin Frequency-Selective Lattices Integrated in Novel Compact MIC, MMIC, and PCA Architectures. IEEE Transactions on Microwave Theory and Techniques, vol. 46, pp. 1936-1948, November, 1998.

[3] I. Bardi, R. Remski, D. Perry, and Z. Cendes.Plane Wave Scattering from Frequency Selective Surfaces by the Finite Element Method. Compumag Conference Proceedings, Evian France, July 2001

[4] A.D.Chuprin,E.A.Parker and J.C.Batchelor.Resonant frequencies of open and closed loop frequency selective surface arrays.Electronics Letters,vol. 36,pp.1601-1603,September 2000. 